\title{
MASSIVE HAEMOPTYSIS FROM ARTERIO-BRONCHIAL FISTULA IN PULMONARY TUBERCULOSIS
}

\author{
BY \\ M. BERARD AND JAUBERT DE BEAUJEU \\ Lyons
}

(RECEIVED FOR PUBLICATION FEBRUARY 25, 1952)

While the pathogenesis of haemoptyses in tuberculous patients is often obscure and subject to the most diverse interpretations, there are some instances in which the mechanism of the haemorrhage can be identified by the presence of clear anatomical and pathological findings. We have found it possible to distinguish a certain number of particular types of haemoptysis corresponding to definite anatomical lesions such as Ameuille, Gautier, and Iglesias-Bettancourt (1936) reported when they described direct communication between a vessel and a lobar bronchus secondary to a parenchymatous ulcerative process. Similarly in necropsies upon patients dead from violent haemoptyses fistula formation between a mediastinal vessel and a major bronchus can sometimes be demonstrated.

Pulmonary resection has given us the opportunity, not only of appreciating this process, but also of understanding the pathological changes in the hilum of the lung that lead to such a complication, and the role played by the lymphatic system in its origin.

In 1931 Arnstein published 10 case histories of elderly patients dead from haemoptyses in whom necropsy revealed fistula formation between the lower end of the trachea or one of the stem bronchi and a major mediastinal vessel. In one of these the aorta had ruptured into the lower end of the trachea by the agency of a histologically tuberculous lymph node, while in the others investigation showed that the fistula followed ulceration of a pulmonary vessel. In all, the presence of a lymph node in contact with the fistula was described. Only in one case was it possible to establish histologically the tuberculous nature of the node, but in all the macroscopic appearances of the adenopathy, very different from that of the anthracotic glands found in old subjects, favoured a tuberculous origin. It was by the process of caseation in the gland, an actual tunnelling between bronchus and artery, that the fistula had been created.
Arnstein made the following observations. HoN pointed out that the mechanism by which haemor-? rhage is arrested is by the formation of a thrombus in the fistulous communication itself; and this, $c$ he said, explained the intermittent character of the haemoptyses. He emphasized the difficulty in $\overrightarrow{0}$ making such a diagnosis clinically, especially when chest radiographs showed the presence of a parenchymatous lesion which might, by itself, haveo explained the haemoptysis. Arnstein's observations concerned fatal haemoptyses in which anatomica proof could be brought forward in support of $a \frac{\mathrm{D}}{\mathrm{D}}$ specific pathogenesis for the haemorrhages.

In the cases we have observed, and in which weo have been able to confirm during operation the same findings described by Arnstein, that is to say a direct communication between vessel and bronchus by the agency of a lymph node, we found a charac- teristic clinical picture, that of massive recurrent haemoptyses.

A case was reported by Soave (1950) of inter mittent fistula occurring between a branch of thein. pulmonary artery and the middle lobe bronchus $\frac{0}{3}$ His patient, aged 48, had had recurrent haemoptyses of varying degree since the age of 31 , some milde and transitory, but others taking on a dramaties character as much from their abrupt onset as from their abundance. Bronchoscopy revealed a stenosisos of the middle lobe bronchus, although the presencer of such a lesion did not explain the severity or then repetition of the haemoptyses, and it was their ${ }^{\omega}$ alarming character that, in itself, indicated surgery The operation, carried out in January, 1948, involved? a right pneumonectomy owing to the inability of the surgeon to perform a middle lobectomy since the state of the hilum, profoundly involved in the old inflammatory process, rendered dissection impossible. Examination of the specimen showed a direct communication between the middle lobe bronchus and artery. A thrombus obliterated the orifice of the defect. Only on microscopy was if possible to identify inflammatory tissue in which 
elements of lymphatic origin predominated in the region of the fistula.

The findings at operation in two of our patients resembled in certain respects the case described by Soave.

\section{Case Reports}

Case 1.-This patient, a woman aged 26 , had a clinical history going back six years. In 1943 an ulcerative tuberculous lesion had been discovered at the apex of the right lung. The pleura being adherent, an extrapleural pneumothorax was established in December, 1943. A purulent effusion succeeded this operation, which, owing to the inadequacy of aspirations and lavage, required open drainage in May, 1944. From then on a series of operations designed to obliterate the suppurating cavity was performed. From 1944 to 1947 two saucerizations of the pocket, three thoracoplasty stages, and a partial scapulectomy were carried out. By December, 1947, the pleuro-cutaneous track had closed. But the sputum was still positive and tomography revealed a small residual cavity, and the patient remained in hospital until the beginning of 1949. In June, 1949, the first haemoptysis suddenly occurred. It was violent, sudden, and profuse, and was neither preceded nor accompanied by cough or by a febrile reaction. For a month, and in spite of treatment, small haemorrhages occurred each day. The persistent bleeding, which was attributed to the residual cavity seen on previous tomography, was regarded as an indication for resection.

When the patient was admitted to the surgical wards all investigations were negative, on tomography as well as on successive sputum examinations. Bronchoscopy showed on the right the mucosa much reddened but no stenosis or any specific mucosal lesions. The right lung was well collapsed; the left was healthy. Under these conditions operation was put off, since no sufficient justification for it could be found. The patient was kept under observation for three months. Her temperature remained normal; her general condition remained satisfactory; she neither coughed nor spat; lavages were negative. Nonetheless, from time to time she had little haemoptyses of an absolutely ordinary kind for which radiography and the most detailed tomograms revealed no cause.

At the end of October, 1949, another dramatic haemoptysis occurred, this time extremely profuse and estimated at more than a litre. The gravity of this episode, during which the patient's life was in danger, made us decide to operate.

The operation was performed on November 22, 1949 , under general anaesthesia after intubation with pentothal and curare. Thoracotomy was achieved by resecting the anterior segment of the seventh rib, then division upwards through the three intercostal spaces above. On opening the thorax the lung was found completely but loosely adherent, and was easily freed. The lower lobe siemed to be normal; the upper was small and atelectatic; the middle lobe was normal. In these circumstances, as only the upper lobe appeared abnormal, it was decided to do an upper lobectomy. A start was made at the hilum; it was enveloped in dense fibrous tissue with several inflamed lymphatic nodes. To begin with, the superior vein was dissected and tied; beneath it the artery to the upper lobe was identified and dissection of this was begun. This proved to be difficult, especially behind, where the artery was wholly plastered against the upper lobe bronchus. In the course of this dissection a small tear was made in the side of the artery, but did not prevent us continuing to clear the vessel and putting a ligature round its base. It was then realized that the segment of the artery situated distal to the ligature was not only adherent to the upper lobe bronchus in an extremely intimate fashion, but was fused with it. In an effort to separate the two structures the bronchus was inadvertently opened, and it was seen that at the point of fusion there was an actual loss of substance in the bronchial wall, and the lumen of the artery opened directly into the bronchus. The two elements were inseparable without tearing the artery: it was a true arterio-bronchial fistula. The whole operative field at this point was obscured in very dense cellular tissue with a number of small lymphatic nodes.

The upper lobe bronchus was clamped above the fistula, flush with the stem bronchus, closed by interrupted 0000 silk sutures, and covered with pleura. The thorax was closed after a small axillary drain had been inserted.

The upper lobe of the operation specimen was tiny and atelectatic. On section, two small nodules full of thick caseation were found; these corresponded to the original anterior cavity which had closed under the thoracoplasty. We submit that there can be no doubt that the massive haemoptyses suffered by this patient originated from the arterio-bronchial fistula.

The post-operative course was afebrile and straightforward. The patient's general condition rapidly improved and she gained weight. There were no more haemoptyses and respiratory function notably improved; there was less dyspnoea on effort. We do not know how to explain her functional improvement. At present, more than two years after operation, recovery is perfectly maintained.

Case 2.-The history of this patient, a woman, goes back to October, 1943, at which time tuberculous infiltration was found at the left apex. An attempted artificial pneumothorax failed, and sanatorium treatment was instituted. In April, 1945, an upper left thoracoplasty was done and led to complete recovery, and by July, 1950, the sputum was negative. In August, 1950, haemoptysis of about a glassful of red blood occurred, and this was twice repeated. The temperature rose to $38.7^{\circ} \mathrm{C}$. At this point the patient was given $20 \mathrm{~g}$. streptomycin and sent to the surgical side. On admission she was in fairly good general condition. There was a little sputum (five or six expectorations a day); the temperature swung between $37.2^{\circ} \mathrm{C}$. in the morning and $38^{\circ}$ at night. Radiographs showed no pulmonary lesions under the left thoracoplasty, but at the base some diffuse small nodules. The right lung was normal. Tomographs showed no cavity. 
Operation was performed on September 26, 1950. General anaesthesia was used after intubation under pentothal and curare. Thoracotomy by resection of the sixth rib was performed. The lung was completely adherent; the lower lobe was relatively well aerated; the upper lobe was small and atelectatic. Since tuberculous infiltration could be felt in the lower lobe an upper lobectomy could not be contemplated and a start was made to free the upper lobe from the summit of the thorax, to which it was very adherent, in order to see the hilum. On approaching the hilum it was found to be infiltrated and on palpation one could feel a bulky lymph node extending into the mediastinum. The vagus was engulfed in the process, and it was impossible to separate the bronchus. A search was made for the pulmonary artery and it was found closely fused to the stem bronchus. All attempts to free the two structures proved impossible; and, because of the previous massive haemoptyses, it was thought likely that another arteriobronchial fistula of the stem bronchus had to be dealt with. Since it was impossible to tie the artery directly it was decided to approach it intrapericardially, and after injecting novocaine the pericardium was opened. The peribronchial fibrosis had so retracted the left pulmonary artery that it was only possible to ligate it flush with the bifurcation of the main pulmonary trunk. After division an organized clot was found in its lumen obliterating the fistula between it and the bronchus. In fact, after this clot had been removed the bronchus lay open, and blew in the operation field. The bronchial wall had been completely infiltrated, bound up in the midst of a bulky adenopathy which rendered it particularly difficult to clamp the bronchus after its root had been dissected out. The operation was completed by intrapericardial ligature of the two pulmonary veins. Before this we felt considerable anxiety because of the open bronchus and the consequent trouble with oxygenation and blood leakage into the bronchial tree, but as soon as the state of the patient seemed satisfactory the dissection of the stem bronchus was completed. This was carried far up, but without finding healthy bronchial wall. Nonetheless the bronchus was efficiently sutured close to the carina. The stump was not pleuralized and the chest was closed after a De Pezzer catheter drain had been inserted. The upper lobe of the operation specimen was atelectatic. At its apex were two or three calcified foci, but no residual cavity, and some pneumonic nodules were present in the lower lobe. The stem bronchus was pathological, and between it and the trunk of the pulmonary artery there was a fistula. The walls of the artery and the stem bronchus were intimately fused; in fact they merged into one another. The fistulous orifice itself measured 1 to $2 \mathrm{~mm}$. and no lymphatic tissue at the actual site of the fistula could be found microscopically.

Delirium and crises of excitability followed immediately upon the operation and lasted eight days. From the pulmonary point of view a little congestion at the right base occurred without shortness of breath; this resolved on the seventh day. On October 8, when her mental and general state had for the past three days been much better, the patient suddenly collapsed and die during a meal. Whilst the actual diagnosis could not verified, we felt no doubt that death was due to pulmonary embolus.

These two cases allow us to make certa周 comments. Without doubt the pathogenesis of these haemoptyses lies in the direct communicatio of the main trunk of the pulmonary artery, or its upper lobe branch, with the stem bronchus in the one case, and the upper lobe bronchus in the other The bronchial mechanism of the bleeding was different from that described by Fleischner (1937 7 and Cordier and Mounier-Kuhn (1938) under the term haemorrhagic tracheo-bronchitis. According to these authors, a congestive reaction of the bronchial mucosa occurred opposite the point where a diseased node was in contact, and of bronchoscopy a hyperaemic congestive zone the mucosa could clearly be seen, situated mosf often at the level of the carina or at the origin of one of the stem bronchi. Furthermore it is rare in these cases for bleeding to be profuse: the haemळptysis is often confined to blood in one or tw expectorations.

In our two cases the bronchoscopic examination did not provide us with precise information. was noted, in our first observation, that the mucosa of the right upper lobe bronchus was red and congested. In view of what we found at operation it will readily be understood that the lesion situated in the right upper lobe bronchus could not be identified by direct vision, and even the rights angled telescope did not furnish us with accurate information because of the hyperaemic state of the mucosa. It seems to us that it is essentially the particular clinical character of haemorrhages from an arterio-bronchial fistula which one must bear mind. What are the notable characteristics of suc haemoptyses as seen in our two cases? In the fir case, especially, it was the circumstance of massive haemorrhage occurring in a tuberculous patiejo clinically and radiologically well. In fact, after tho multiple operations she had undergone-extrat pleural drainages, thoracoplasties, partial scapt: lectomy - the collapse of the right apex was as complete as possible, and no residual cavity coula be demonstrated either by ordinary radiography by tomograms. Furthermore, the various sputum examinations (both direct and after lavage proved negative. In our second patient tuberc度 bacilli had never been demonstrated in all th sputum examinations carried out since the thoraco plasty. No residual cavity had been seen either of straight films or tomography, nor was a cavity foung in the specimen removed at operation. 
The nature of these haemorrhages was, we submit, typical. They were notable for their sudden onset, their violence, and their volume. They appeared with no warning, and no premonitory fits of coughing. The blood was not frothy, and they stopped as abruptly as they began, without that " tailing off" classically seen in bleeding from polyps and bronchial adenomas. All these features suggest haemoptysis of bronchial origin rather than bleeding from the parenchysa of the lung. How can their pathogenesis be explained? As Arnstein (1931) has demonstrated they result from fistula formation between a bronchus and a branch of the pulmonary artery by the agency of a lymph node. And how, in these circumstances, is their intermittent character explained? Undoubtedly the mechanism suggests a fair analogy with some bleeding gastric ulcers in which a large vessel becomes eroded. The arterial "fistula " may close by the formation of a thrombus; and perhaps also there may be some element of reflex vascular spasm touched off by the flooding of the bronchus.

While the existence of such haemorrhages is striking enough in the cases reported above, the operative findings in a number of our other resections for tuberculosis give us an insight into the anatomical manner in which they occur.

It is often the case in old disease, especially where there is a tuberculous stenosis of the stem bronchus, that one finds a number of bulky lymph nodes in the hilum. For further information on this point we reviewed the operative findings in nearly 100 resections for stenosis. Twelve of these showed intimate cohesion of a branch of the pulmonary artery either to the stem or lobar bronchus. In one instance there was a lymph node between artery and bronchus which had ulcerated into the walls of both in such a way that they could not be separated without being torn. In a number of others the anatomical relations, though less intimate, were no less close, and one can see how caseous breakdown in a node, or suppuration during an intercurrent infection, might cause a fistula to form.
The interest of our two cases lies in their having clearly proved to us that arterio-bronchial fistula is a true entity. We consider it a diagnosis which must always be thought of in the presence of sudden and violent haemoptyses occurring in apparently stabilized tuberculous patients who present no radiological evidence by which they might be explained. We also think such a presumptive diagnosis an absolute indication for radical surgery.

Finally we emphasize the technical difficulties these operations may present: first with dissection of the hilum, then with the dissection and separation both of the pulmonary artery and bronchus. In the face of such difficulties there should be no hesitation in using the intrapericardial route, and it was only by employing this route in our second case that we were able safely to achieve satisfactory haemostasis.

\section{SUMmaRY}

Two cases are reported in which an arteriobronchial fistula was discovered in the course of operation upon tuberculous patients. In the first there was a fistula between the artery and bronchus to the right upper lobe; in the second the main trunk of the pulmonary artery itself communicated with the left stem bronchus. These two patients presented with violent, profuse, and repeated haemoptyses whose gravity dictated pulmonary resection.

The presence of haemoptyses of characteristic type-sudden, often profuse, of non-frothy bloodoccurring in a patient suffering from pulmonary tuberculosis, apparently stabilized, or who has undergone effective surgical collapse therapy, should suggest that an arterio-bronchial fistula may exist.

Pulmonary resection provides, under these circumstances, a cure.

\section{REFERENCES}

Ameuille, P., Gautier, J., and Iglesias-Bettancourt (1936). Bull. Soc. méd. Hôp. Paris, p. 204.

Arnstein, A. (1931). Beitr. Klin. Tuberk., 78, 55.

Cordier, V., and Mounier-Kuhn, P. L. (1938). Pr. méd., 46, 1065.

Fleischner, F. (1937). Wien. klin. Wschr., 50, 929.

Soave, F. (1950). Thorax, 5, 90. 